\title{
WITH HEAD AND HEART AND MIND: THE LIFE AND LEGACY OF ARCHIE COCHRANE
}

\author{
A Smith, Consultant Anaesthetist \\ Royal Lancaster Infirmary
}

\section{INTRODUCTION}

These days, evidence-based medicine is firmly on the minds of clinicians and policy-makers. Similarly, a critical attitude to the provision of health services in general is not uncommon. It was not, however, always so. This year, the book Effectiveness and efficiency: random reflections on health services by Archie Cochrane has been re-issued. I remember reading it as a student, although it was then already over ten years since its first publication. Re-reading it, I found it had influenced me more than I had realised. In the light of the continuing debate over evidence-based medicine, and as the Cochrane Collaboration grows apace, I was stimulated to look again at Cochrane's life and how it shaped his ideas.

\section{A LIFE LESS ORDINARY?}

Archie Cochrane was born in Galashiels in 1909. After reading natural sciences at Cambridge, he studied medicine in London, graduating in 1938. One of his student years was spent in the International Brigade during the Spanish Civil War; later he returned to medical research and then joined the Royal Army Medical Corps in 1940. He was captured in Crete in 1941 and spent some years as a prisoner of war in various parts of Europe.

After the war, he conducted meticulous and painstaking epidemiological fieldwork on pneumoconiosis among the mining communities of the Rhondda Fach, and became Professor of Chest Diseases at the Welsh National School of Medicine. In 1969 he became fulltime director of the MRC's epidemiology unit, focusing particularly on randomised controlled trials aimed at discovering the optimal place of treatment and the optimal length of hospital stay. In 1971 the Nuffield Provincial Hospitals Trust invited him to deliver its annual Rock Carling lecture, which Cochrane used to elaborate on this and other applications of epidemiology to clinical practice. He became the first President of the Faculty of Community Medicine in the same year. He remained active in research and continued to travel widely until shortly before his death in 1988 .

\section{EFFECTIVENESS AND EFFICIENCY}

When Effectiveness and Efficiency first appeared, few could have foreseen the effect it was to have on healthcare throughout the world. Cochrane himself remained vaguely surprised and somewhat dismayed by the attention it inspired, feeling that it overshadowed what he considered his more important work on the epidemiology of chest diseases in the 1950's. The book, now available again through the Royal Society of Medicine Press, is a quirky and self-effacing blend of anecdote and science, logic and passion, which says more in its 90 -odd pages about the problems and challenges facing modern medicine than textbooks and journals ever did. Cochrane defined his terms carefully. He observed that, if we are to get the best from the National Health Service, we must be able to measure benefit as well as cost. He saw that, in order to make this practically possible, we must be able to measure the effect of a particular medical action on the natural history of disease. This he called effectiveness. We need also to determine the optimum use of personnel and materials in achieving these results. This he called efficiency. Immediately he recognised that there were problems in applying this approach to much activity of the NHS, because although these two indices could be used for assessment of therapy (attempts at cure) they could yield no information on the health service's other main function, that of providing, in his words, "board and lodging and tender, loving care." His third index, equality, referring mainly to equality in these non-therapeutic aspects of hospitals, for some reason never featured in the book's title, but pervades the work nonetheless.

Cochrane's wartime experiences as a medical officer in prisoner of war camps seem to have affected him greatly. On the one hand, he was amazed to see, despite severe epidemics of typhoid, diphtheria, jaundice and sand-fly fever, with aspirin, antacid and antiseptic as the only medications available, that few of his patients died. This demonstrated, he wrote, "the relative unimportance of therapy in comparison with the recuperative power of the human body", and coloured his view of the evaluation of new treatments. On the other hand, certain events seem to have helped to form his later view that care and compassion are all the more important when no effective therapy is available. His experience of attending a dying Russian soldier is recounted in his autobiography: "The ward was full, so I put him in my room as he was moribund and screaming and I did not want to wake the ward. I examined him. He had obvious gross bilateral cavitation and a severe pleural rub. I thought the latter was the cause of the pain and screaming. I had no morphia, just aspirin, which had no effect. I felt desperate. I knew very little Russian then and there was no one in the ward who did. I finally instinctively sat down on the bed and took him in my arms, and the screaming stopped almost at once. He died peacefully in my arms a few hours later. It was not the pleurisy that caused the screaming, but loneliness. It was a wonderful education about the care of the dying."

A later chapter of the book deals with input to, and output from, the NHS. Cochrane pointed out that, for most therapies, 
a convincing relationship between input and output had not been demonstrated, and chose instead to examine the NHS as a whole. Using the large increase in numbers of health service staff since the inception of the NHS as evidence of increased input, he took changes in life expectancy and (somewhat impishly) certified time 'off sick' as markers of death and disability in the population. He showed that there had been little change in overall life expectancy (falling mortality rates for $\mathrm{TB}$, for instance, being balanced by rising rates for ischaemic heart disease), and that certified sickness increased slowly but surely between 1954 and 1967! By selecting these examples, Cochrane creates a vague unease in the mind of the reader - like any good salesman, he creates dissatisfaction amongst his audience with the current state of affairs, making them more receptive to his suggestions for improvement.

He goes on in later chapters of the book to discuss evaluation of evidence, favouring randomised controlled trials over observational studies, which, he argues, are themselves preferable to 'clinical opinion'. This has modern echoes in the 'hierarchy of evidence' commonly seen today (Figure 1). There follow examples of prevention, diagnosis and treatment to illustrate Cochrane's argument on the desirability of proper evaluation of what has now come to be known as 'health technology'.

\section{Level I Systematic review of randomised controlled trials \\ Level II Single randomised controlled trial \\ Level III Case-control study \\ Level IV Observational study \\ Level V Case series \\ Level VI Single case report \\ Such hierarchies give a measure of how soundly a particular intervention is based. For instance, one can be much more confident that a therapy proven in a randomised controlled trial is effective than one whose evidence of effectiveness comes from a retrospective case series}

Figure 1 Typical hierarchy of evidence

Effectiveness and Efficiency is often acknowledged to be the start of the whole 'evidence-based medicine' movement. As I have tried to emphasise, however, the unfeeling application of science was never Cochrane's aim. The concept of equality reappears towards the end of the book, referring particularly to equality of hospital environment. He drew attention to the discrepancies in 'quality of life' between acute hospitals and long-stay institutions. In his conclusions he went so far as to suggest that the money which he thought could be saved by the proper application of effective therapies should be diverted to improve the lot of the many NHS patients for whom cure was not possible and whose lives were influenced most by the quality of care they received. The reissued version contains a codicil of Cochrane's reflections on the reaction to the book's publication, in which he defined quality quite simply: "the really important factors are kindliness and ability to communicate on the part of all members of the medical team".

He went on: "We attempt to teach medical students psychology and sociology, but will we really make them kindlier? We desperately need a test which we could apply to aspirant medical students which would tell us whether they would remain kindly in middle age; but I am advised that the development of such a test is very improbable".

\section{COCHRANE'S CHALLENGE}

In a further publication in 1979, Cochrane wrote: "It is surely a great criticism of our profession that we have not organised a critical summary, by specialty or subspecialty, adapted periodically, of all relevant randomised controlled trials".

In the same essay, he singled out obstetrics (whether rightly or wrongly) as the medical specialty which had made the least effort to seek good evidence on which to base its practices. This rebuke encouraged an international collaborative effort which was established to prepare systematic reviews, using the statistical technique of metaanalysis when appropriate, of all the randomised controlled trials relevant to care during pregnancy and childbirth. This began to yield results in the late 1980's, the findings being published as the book Effective Care in Pregnancy and Childbirth and electronically as the Oxford Database of Perinatal Trials. Such systematic reviews began to be used in healthcare decision-making and clinical practice, and were also taken up enthusiastically by lay people. (It has been said that, in the early 1990 's, local co-ordinators of the National Childbirth Trust were more likely to be referring to the Database of Perinatal Trials than to the obstetricians with whom their members were booked.) The encouraging reception given to this first wave of collated evidence on effectiveness in healthcare coincided with the introduction of the NHS Research and Development Programme in 1991, and Cochrane's idea was favoured within it.

\section{THE COCHRANE COLLABORATION}

In October 1992 the world's first Cochrane Centre opened in Oxford. Within a year it was clear that this was destined to become a worldwide venture, and its name had to be changed to the UK Cochrane Centre. In October 1993, 77 people from nine countries founded the Cochrane Collaboration. Since then, thirteen other Cochrane Centres have been opened throughout the world, to support local researchers and act as points of contact.

Collaboration - by internally and externally fostering good communications, open decision-making and teamwork

Building on the enthusiasm of individuals - by involving and supporting people of different skills and backgrounds

Avoiding duplication - by good management and co-ordination to maximise economy of effort

Minimising bias - through a variety of approaches such as scientific rigour, ensuring broad participation, and avoiding conflict of interest

Keeping up to date - by a commitment to ensure that Cochrane Reviews are maintained through the identification and incorporation of new evidence

Striving for relevance - by promoting the assessment of healthcare interventions using outcomes that matter to people making choices in healthcare

Promoting access - by wide dissemination of the outputs of the Collaboration, taking advantage of strategic alliances, and by promoting appropriate prices, content and media to meet the needs of users worldwide

Ensuring quality - by being open and responsive to criticism, applying advances in methodology, and developing systems for quality improvement

Figure 2 The principles of the Cochrane Collaboration

Now the Cochrane Collaboration is a truly international organisation whose aims are simple: to help people make 
well-informed decisions about health by preparing, maintaining and disseminating systematic reviews of the effects of healthcare interventions. Its eight guiding values are shown in Figure 2. Preparation and maintenance of the reviews is co-ordinated by Collaborative Review Groups. Currently there are over 40 groups registered, covering areas as diverse as stroke, injuries, infectious diseases, ENT and schizophrenia. Not all areas of healthcare are represented yet and clearly the output of the Collaboration reflects the interests of the groups that have come forward.

Cochrane reviews are published electronically on the Cochrane Library. This is available on CD-ROM, floppy disk and online. As well as the reviews, the Library also contains the Controlled Clinical Trials Register (a database of 224,319 trials, some either unpublished or not identified on Medline), a large collection of references on review methodology, and the Database of Abstracts on Effectiveness (material not prepared by Cochrane groups but still meeting certain quality standards). The CD-ROM is published quarterly, and the last issue contains 576 complete reviews and 538 protocols for future reviews.

\section{WHERE TO NOW?}

The Collaboration has made an encouraging start, particularly in terms of recruitment and support. Clearly it has inspired many people and channelled their enthusiasm. Governments throughout the developed world have been quick to support work on clinical effectiveness, though it is impossible to say whether showing what works and what doesn't work in medicine will save money. Cochrane himself was not necessarily interested in saving money, suggesting instead that it be redistributed to improve care for NHS patients, especially the longterm inmates of unglamorous institutions.

The widespread introduction of information technology has also contributed to the Collaboration's development. The creation of electronic bibliographic databases has simplified the task of searching for relevant material for reviews (as anyone old enough to remember Index Medicus will agree) whilst the rise of the personal computer allows easy updating and rapid dissemination of reviews. A further factor is the close involvement of healthcare consumers within Collaborative Review Groups, selecting review topics and outcomes of importance, commenting on protocols, and making use of the finished reviews as active participants in decision-making about their health.

As the Collaboration grows, however, areas for improvement can be discerned. For instance, the Collaboration is still biased towards the needs of the developed world, both in the location of Centres and in the material reviewed. Furthermore, just as there is inequality of health between developed and developing countries, so there is a dearth of both research skills and the means of accessing potentially useful Cochrane reviews within poorer nations. Many medical schools in Africa may have only one computer, often obsolete by Western standards, and may not be able to use the Cochrane Library (assuming that they can afford to buy it). To try to get round this, the World Health Organisation has incorporated some of the most relevant Cochrane reviews onto a single floppy disk, mailing copies free of charge to anyone who asks for one, and giving out copies to visitors from developing countries to its Geneva headquarters.

A further remaining challenge is to ensure that the findings of reviews are put into practice, and do not just stay, unused, on the Cochrane Library. However, one of the strongest reasons why Cochrane reviews may not find their way into clinical practice is applicable to 'evidence-based medicine' in all its guises. This is the simple fear of some clinicians that their clinical judgement will be supplanted and their patients enslaved to an impersonal 'cookbook' approach. As far as I can see, this was never intended; Cochrane reviewers are urged, in fact, simply to present the evidence as they see it (with possible biases made plain) rather than to take their role a stage further and tell their readers what they should do in practice.

Healthcare workers must still exercise their judgement as to how and when the results should be applied, and nothing can replace the human touch. I am sure that the last thing Cochrane would have wanted was a new orthodoxy where dogma took precedence over common sense and, more importantly, common kindness.

\section{FURTHER READING}

Cochrane A Effectiveness and Efficiency: Random Reflections on Health Services. London: Royal Society of Medicine Press, 1999 (first published 1972 by Nuffield Hospitals Provincial Trust)

Maynard A, Chalmers I Non-random Reflections on Health Services Research. London: BMJ Publishing Group, 1997

Sackett DL, Rosenberg WMC, Muir Gray JA, Haynes RB, Richardson WS Evidence-based medicine: what it is and what it isn't Br Med J 1996;312:71-2

Andrew Smith has contributed to the Cochrane Collaboration since 1995 as a handsearcher of journals, translator and reviewer and has been invited to act as an editor in the recently-formed Anaesthesia and Critical Care Group 